\title{
Feasibility study of laser lock-in thermography for micro-crack detection on dental tooth
}

\author{
by Andrew Ngo*, Vinod Kumar*, Kenneth Low **, Brian Stephen Wong** \\ * Institute of Materials Research and Engineering, 2 Fusionopolis Way, Innovis, \#08-03, Singapore 138634 \\ ** Nanyang Technological University, N3.2-01-30, 50 Nanyang Ave, Singapore 639798 \\ Corresponding author: ngocya@imre.a-star.edu.sg
}

\begin{abstract}
The timely and proper diagnosis and treatment of microcracks in teeth is of paramount importance to improve the tooth prognosis. Early diagnosis of a crack in a tooth and its early treatment is vital to prevent further splitting. In this work, we consider the feasibility study of using visible laser for lock-in thermography for micro-crack detection on artificial dental tooth. Analysis of the findings and results will also be presented.
\end{abstract}

\section{Introduction}

The timely and proper diagnosis and treatment of microcracks in teeth is of paramount importance to improve the tooth prognosis. Early diagnosis of a crack in a tooth and its early treatment is vital to prevent further splitting. Even though various treatment options for tooth fracture had been developed over the past decade [1-3], dental practitioners still experience difficulty in performing a diagnostic evaluation of micro-cracks. This is mainly due to limitations in the available and commonly used examination methods. Needle inspection, fracture staining, dental light microscopy and radiography are the most commonly used diagnostic techniques. However, these techniques possess inherent limitations such as exposure to radiation, resolution limits and visual limits. Therefore, an alternative method for dental inspection that can overcome these limitation for clinical application is sought. Infrared thermography (IRT) is a non-contact, non-destructive testing (NDT) technique that is capable of obtaining images in a relatively short time over a reasonably large area. Although previous work on dental inspection using laser lock-in thermography has proven to be effective with good sensitivity and resolution [4], there exists only a handful of publications in this area. Therefore, the aim of this work is to report the feasibility study of laser lock-in thermography for micro-crack detection on dental tooth.

\section{Experiment}

\subsection{Samples}

The samples used in this experiment are artificial teeth from Shofu Inc. Shofu Inc is a manufacturer and supplier of high quality artificial teeth that assures predictable performance, life-like and natural aesthetics and composite resin that mimics the shades, reflectivity and emissivity of real tooth. As seen from Fig. 1 below, one set of Anterior LIVERA Alpha$\mathrm{CH}$ HT6 teeth and one set of M32 type posterior teeth with A3.5 shade was chosen.

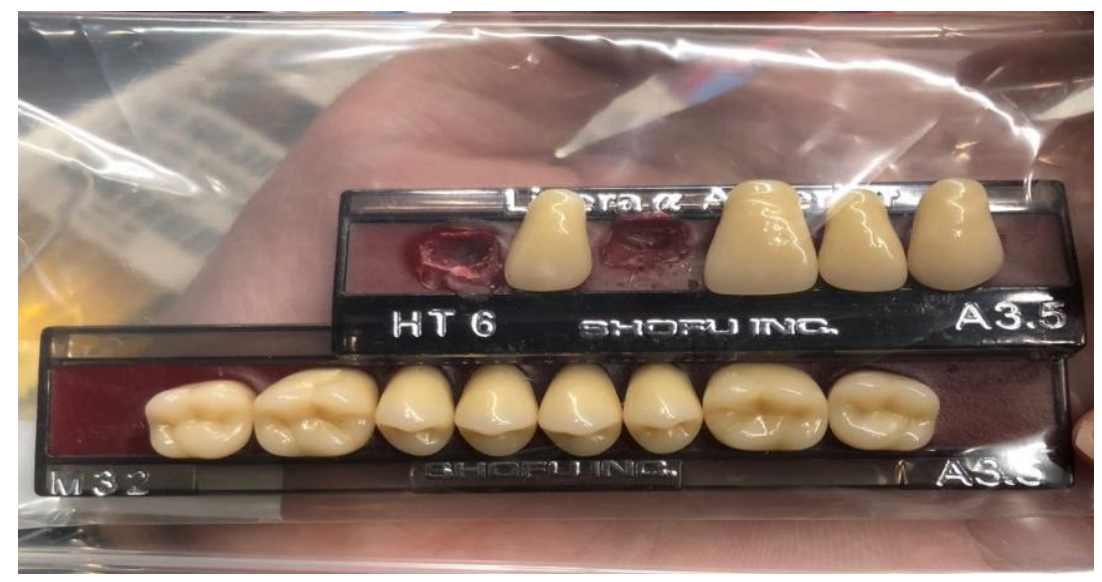

Fig. 1: Shofu Inc - Livera Alpha - CH HT6 Teeth 


\subsection{Set-up}

Fig. 2 shows the set-up used for this experiment. Due to space constraint, the following set-up was chosen instead of a linear one.

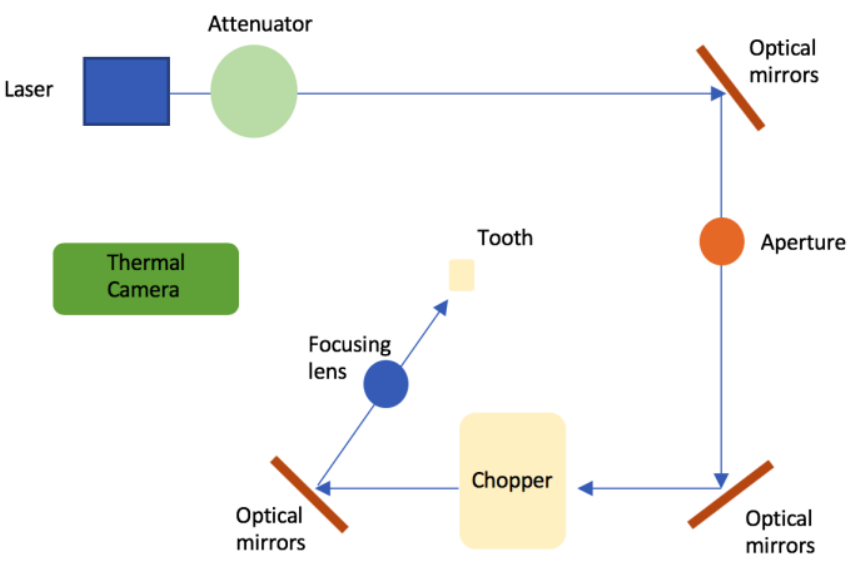

Fig. 2: Layout of the experiment

\section{Results}

Fig. 3 depicts the amplitude image of laser spot on scratch (left) and the intensity profile across scratch (right). As the amplitude might not show obvious signs of disruption of heat propagation, the presence of a defect is not immediately clear via observation. Therefore, in this case, an intensity profile is showed to confirm the presence of a crack, which is denoted by a noticeable spike on the intensity profile.
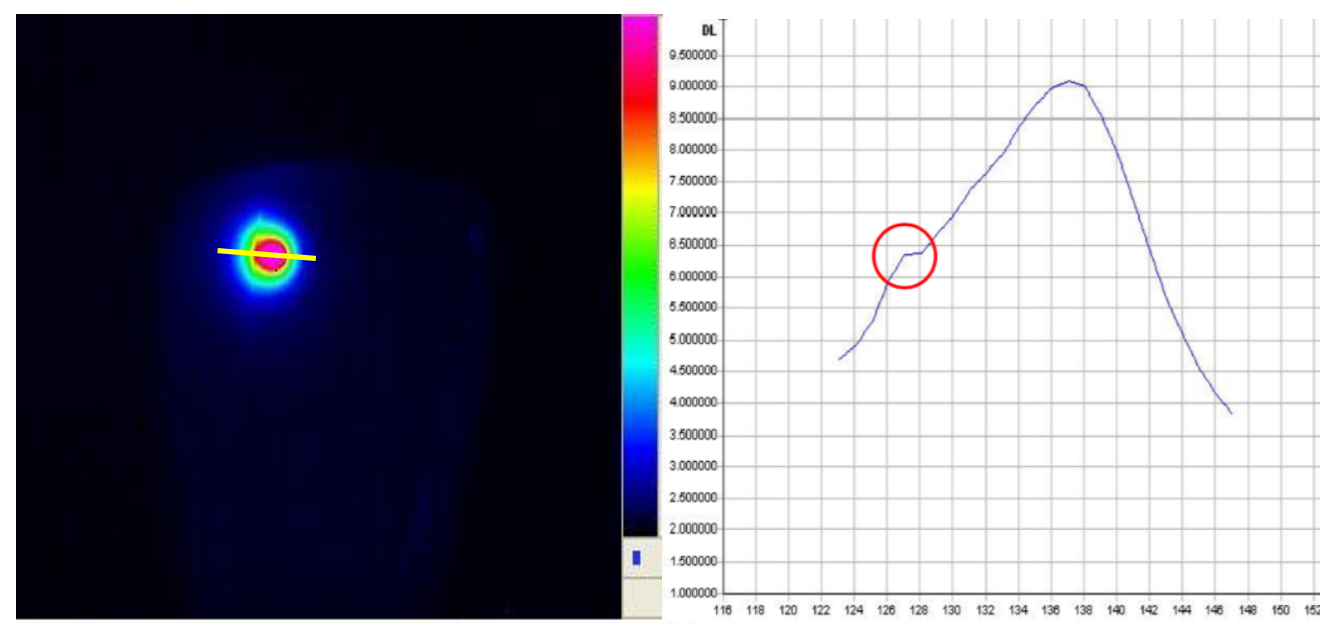

Fig. 3: (Left) Amplitude image of laser spot on scratch; (Right) Intensity profile across scratch

\section{REFERENCES}

[1] S. Oliet, "Treating vertical root fractures", J. Endod. 10 (1984) 391-6.

[2] M. Hayashi, Y. Kinomoto, M. Miura, I. Sato, F. Takeshige, S. Ebisu, "Short-term evaluation of intentional replantation of vertically fractured roots reconstructed with dentin-bonded resin", J. Endod. 28 (2002) 120-4.

[3] O. Niek, R. Joost, L. Bas, B. Ewald, "Seven-year clinical evaluation of painful cracked teeth restored with a direct composite restoration”, J. Endod. 34 (2008) 808-11. 